\title{
Neural Circuitry Underlying Rule Use in Humans and Nonhuman Primates
}

\author{
Silvia A. Bunge, ${ }^{1}$ Jonathan D. Wallis, ${ }^{2}$ Amanda Parker, ${ }^{3}$ Marcel Brass, ${ }^{4}$ Eveline A. Crone, ${ }^{1,5}$ Eiji Hoshi, ${ }^{6}$ and \\ Katsuyuki Sakai ${ }^{7}$ \\ ${ }^{1}$ Department of Psychology and Center for Mind and Brain, University of California at Davis, Davis, California 95616, ${ }^{2}$ Helen Wills Neuroscience Institute \\ and Department of Psychology, University of California at Berkeley, Berkeley, California 94720, ${ }^{3}$ Psychology, Brain, and Behaviour, University of Newcastle, \\ Newcastle upon Tyne NE1 7RU, United Kingdom, ${ }^{4}$ Department of Neurology, Max Planck Institute for Human Cognitive and Brain Sciences, D-04103 \\ Leipzig, Germany, ${ }^{5}$ Department of Psychology, Leiden University, 2300 RA Leiden, The Netherlands, ${ }^{6}$ Brain Science Research Center, Tamagawa University \\ Research Institute, Machida, Tokyo 194-8610, Japan, and ${ }^{7}$ Graduate School of Medicine, University of Tokyo, Hongo Bunkyo-ku, Tokyo 113-8654, Japan
}

Key words: prefrontal; rule learning; behavior; cognition; premotor; ablation; imaging; extracellular recordings

Much of our behavior is focused on minimizing or maximizing a particular goal state. For example, animals generally seek to maximize food intake and minimize energy expenditure (Stephens and Krebs, 1986), although some humans strive to achieve the exact opposite goals. Instrumental to goal-oriented behavior is the ability to learn and use rules that specify the most appropriate response under a given set of circumstances. This ability relies on frontal cortex, an expansive region of the brain that interacts with other brain regions to select and plan contextually appropriate responses. Frontal cortical neurons receive and integrate inputs regarding sensory stimuli, previous responses and rewards, and behavioral rules (Asaad et al., 1998; Kim and Shadlen, 1999; White and Wise, 1999; Hoshi et al., 2000) (for review, see Fuster, 2000; Miller and Cohen, 2001). Frontal cortex comprises a number of subregions, many of which appear to contribute to ruleguided behavior in different ways (Bunge, 2004). Figure 1 shows the Brodmann areas (BA) in the human brain that correspond to the frontal subregions that have been implicated in rule-guided behavior in humans and nonhuman primates.

Our mini-symposium at the 2005 Society for Neuroscience meeting brings together systems and cognitive neuroscientists whose research speaks to the neural basis of rule use. Dr. Parker discusses rule-learning deficits in nonhuman primates associated with the disconnection of frontal and temporal cortices. Drs. Hoshi and Wallis present electrophysiological data from nonhuman primates examining the response profiles of neurons in different parts of frontal cortex during rule maintenance and execution. Finally, Drs. Sakai, Brass, and Crone discuss functional magnetic resonance imaging ( $\mathrm{fMRI}$ ) studies in humans designed to examine the blood oxygenation level-dependent activation profiles of specific frontal regions associated with flexible rule use. Collectively, we seek to understand how rules of varying levels of complexity are represented in the brain, ranging from

Received July 15, 2005; revised Aug. 8, 2005; accepted Aug. 9, 2005. Authors are listed in the order in which their contributions appear in this paper.

Correspondence should be addressed to Dr. Silvia A. Bunge, Center for Mind and Brain, University of California at Davis, 267 Cousteau Place, Davis, CA 95616. E-mail: sbunge@ucdavis.edu.

DOI:10.1523/JNEUROSCI.2937-05.2005

Copyright $\odot 2005$ Society for Neuroscience 0270-6474/05/2510347-04\$15.00/0 simple stimulus-reward associations to conditional rules to higher-level task rules.

\section{Reward-based responses}

The ability to learn that a given action is associated with a reward, and to flexibly update this association as needed, relies on orbitofrontal cortex (BA 11). In the monkey, bilateral damage to orbitofrontal cortex (Izquierdo et al., 2004) or a disconnection between the amygdala and the orbitofrontal cortex (Izquierdo and Murray, 2004) disrupts the ability to select a response on the basis of predicted reward outcomes. Furthermore, electrophysiological studies in monkeys have shown that neurons in orbitofrontal cortex and medial prefrontal cortex (specifically, anterior BA 32) encode the payoff (Tremblay and Schultz, 2000; Matsumoto et al., 2003) and costs (Roesch and Olson, 2004) associated with an action. In humans, damage to orbitofrontal cortex impairs performance on a gambling task in which the goal is to maximize money (Bechara et al., 1994); this impairment appears to be related to a deficit in reversal learning or the ability to reverse previous stimulus-reward associations (Fellows and Farah, 2005).

Damage to dorsolateral prefrontal cortex (DLPFC) (BA 9/46) in humans also impairs gambling performance, but the underlying cause of this impairment is likely to be attributable to a deficit in working memory and/or response selection rather than in reversal learning per se (Bechara et al., 1998; Fellows and Farah, 2005). In contrast to orbitofrontal cortex, damage to DLPFC or ventrolateral prefrontal cortex (VLPFC) (BA 44/45/47) in monkeys leaves intact the ability to use low-level, reward-maximizing rules (Dias et al., 1996; Parker and Gaffan, 1998a; Wallis et al., 2001b). However, DLPFC neurons do encode the identity of a predicted reward (Watanabe, 1996) as well as its magnitude (Leon and Shadlen, 1999).

To assess the respective roles of orbitofrontal cortex and DLPFC in reward-based behavior, Wallis and Miller (2003) trained monkeys to perform a simple reward preference task and simultaneously recorded from both of these regions. The monkey had to choose between two pictures associated with different payoffs: namely, the delivery of more or less fruit juice. Neurons in both areas encoded payoff, but neurons in DLPFC also encoded the monkeys' forthcoming motor response to obtain the 
juice. Furthermore, reward selectivity arose more rapidly in orbitofrontal cortex than DLPFC. These results are consistent with reward information entering the frontal cortex via the orbitofrontal cortex, in which it is passed to DLPFC and used to control behavior.

Extending these findings, Wallis and colleagues (Kennerley et al., 2005) have examined how neurons in medial prefrontal cortex integrate information about the number of lever presses to earn the juice reward (cost) and the probability that the reward will be delivered (risk) in addition to payoff. Medial prefrontal neurons, unlike orbitofrontal and lateral prefrontal neurons, encoded all three decision variables, suggesting that medial prefrontal cortex may play an important role in evaluating the costs and benefits of an action.

\section{Conditional rules}

As noted above, lateral prefrontal cortex is not required for responding on the basis of simple stimulus-reward associations. However, this region is important for learning and using more complex rules referred to as conditional rules, whereby case $\mathrm{A}$ requires one response and case $\mathrm{B}$ requires a different response (Murray et al., 2000; Passingham et al., 2000). Parker and Gaffan (1998a) have conducted disconnection studies in monkeys to determine which neural interactions are necessary for conditional learning. This work reveals that conditional learning relies on interactions between prefrontal cortex and inferior temporal cortex, a region involved in representing visual objects. Monkeys with a prefrontal-temporal lobe disconnection are still able to learn stimulus-reward associations but have great difficulty both in applying previously learned conditional rules and in learning to apply the conditional rule to new sets of objects. This loss of flexibility in applying a previously learned rule to new objects can also be seen in delayed matching to sample performance, in which crossed lesions of prefrontal cortex and perirhinal cortex cause a substantial memory deficit (Parker and Gaffan, 1998b).

The most obvious route by which prefrontal and temporal cortices might interact in conditional learning is via the uncinate fascicle, the only direct corticocortical tract connecting the frontal and temporal lobes (Ungerleider et al., 1989). However, transection of the uncinate fascicle has been shown not to affect conditional learning in monkeys (Eacott and Gaffan, 1992). Another possible route of interaction is through the striatum. While examining changes in neuronal selectivity associated with conditional learning in monkeys, Pasupathy and Miller (2005) found rapid changes in caudate neuron selectivity, followed by changes in behavioral performance and prefrontal selectivity. These findings suggest that the striatum rapidly links object representations with specific actions, whereas prefrontal cortex learns the rewarded associations over multiple repetitions.

\section{Rule implementation}

Once an action representation has been selected, distinct sets of selected motor information must be integrated to perform this action. In the case of arm reaching, two major factors are target location and choice of arm. Dorsal premotor cortex (the dorsal part of lateral BA 6) is thought to be involved in integrating these two types of reach-related information, because it possesses cor- ticocortical connections both with DLPFC and with the arm/ digits area of primary motor cortex [BA 4 (Lu et al., 1994; Dum and Strick, 2005)].

Indeed, Hoshi and Tanji (2000) have shown in the monkey that dorsal premotor neurons initially gather information about both the target location and arm use, and that subsequent activity specifies their combination. Thus, dorsal premotor cortex participates in integrating distinct motor information, in addition to preparing future movements (Wise, 1985). Unlike prefrontal neurons, dorsal premotor neurons tend not to be sensitive to the identity of visual objects used for motor instruction (Kurata and Wise, 1988; Mitz et al., 1991; Wallis and Miller, 2003). These and other findings suggest that prefrontal cortex and dorsal premotor cortex play fundamentally different roles in rule-guided behavior. Specifically, prefrontal cortex creates novel information for behavioral selection by processing broad ranges of information while conforming to behavioral rules, whereas dorsal premotor cortex integrates multiple types of motor information generated in prefrontal and parietal cortices and other regions to transform the selected action representation into a behavioral response (Tanji and Hoshi, 2001).

\section{Flexible rule use}

The most challenging aspect of rule use is the need to adapt one's response to a given context when this response no longer yields desirable results. Flexible task switching is generally thought to rely on lateral prefrontal cortex (for review, see Bunge, 2004). However, fMRI and electroencephalographic studies in humans also implicate parietal cortex and presupplementary motor cortex (pre-SMA) (medial BA 6) in task switching (Dreher and Berman, 2002; Sohn et al., 2003; Rushworth et al., 2005), and transcranial magnetic stimulation studies in humans have shown that transient stimulation of parietal cortex or pre-SMA/medial wall leads to a slowing in the ability to switch from one task to another (Rushworth et al., 2001; Rushworth et al., 2002). A recent electroencephalographic study in humans shows that activity in the lateral prefrontal cortex precedes activity in parietal cortex during the updating of task rules (Brass et al., 2005b), supporting the hypothesis that lateral prefrontal cortex provides an abstract task representation that is then transmitted to, and/or further specified in, posterior cortices (Brass and von Cramon, 2004; Stoet and Snyder, 2004).

Bunge, Crone, and colleagues have recently provided fMRI evidence in humans for the dissociability of two components of task switching (Crone et al., 2005). VLPFC was primarily associ- 
ated with retrieval of the currently relevant task rule, whereas pre-SMA/SMA and the basal ganglia (caudate nucleus) were more closely linked to suppression of the previously relevant responses or response contingencies. Parietal activation reflected both rule retrieval and task-set suppression demands. Additionally, fMRI data from participants aged $8-25$ years provide evidence for different rates of functional maturation of these brain regions: adult-like patterns in parietal cortex by age $8-12$ years and in pre-SMA/SMA by age 13-17 years but immature patterns in VLPFC until adulthood (Crone et al., 2004). These findings are broadly consistent with observed developmental trajectories for task switching and rule retrieval.

In addition to the VLPFC and DLPFC regions discussed above, Brass, von Cramon, and colleagues have shown using fMRI in humans that a more posterior region in lateral prefrontal cortex is also involved in rule use (Brass et al., 2005a). This region, for which the underlying cytoarchitectonic structure still has to be determined, is located at the junction of the inferior frontal sulcus and the inferior precentral sulcus and has therefore been termed the inferior frontal junction area. A series of fMRI studies suggests that this region is involved in the environmentally guided updating of task rules (Brass and von Cramon, 2002, 2004). These findings indicate that several prefrontal, parietal, and subcortical structures contribute in different ways to flexible rule use.

\section{Representation of task set}

A task set is a neurocognitive state in which an upcoming task is prospectively configured. It reflects not just which items a subject is preparing to process but also how they plan to process the items or the rules of the to-be-performed task. Sakai and Passingham (2003) have collected fMRI data while subjects performed a task in which they received instructions in advance of the task stimuli. They performed functional connectivity analyses showing that anterior prefrontal cortex (BA 10) activation is closely correlated with that of different prefrontal regions depending on which task the subject is preparing to perform. Anterior prefrontal cortex interacts strongly with a region associated with spatial working memory, the superior frontal sulcus, when subjects prepare to perform a challenging spatial task. In contrast, this region interacts strongly with a region associated with phonological working memory, the posterior VLPFC, when subjects prepare to perform a challenging verbal task.

In an additional study, presented at the Society for Neuroscience annual meeting, Sakai and Passingham asked subjects to make semantic or phonological judgments about words and found that anterior prefrontal cortex interacted with anterior or posterior VLPFC depending on whether the subjects were to perform a semantic or phonological task. Collectively, these data suggest that anterior prefrontal cortex assists in preparing for an upcoming task by coordinating with brain regions that will be needed to perform that task. Additional fMRI research indicates that this region represents high-level rules composed of several lower-level rules, consistent with the hypothesis that VLPFC and anterior prefrontal cortex are hierarchically organized, with the latter integrating across representations held in the former (Bunge et al., 2003; Crone et al., 2005).

\section{Strategy use}

Several recent studies have examined the neural substrates of strategy use: that is, open-ended rules that govern choices at an abstract level, without specifying the appropriate response to a given stimulus. Gaffan et al. (2002) trained monkeys to select specific stimuli either persistently or only sporadically to receive a reward and showed that the use of these strategies, like conditional rules, relies on prefrontal-temporal interaction through a different route than the uncinate fascicle. Neurophysiological studies in monkeys support these findings. For example, prefrontal neurons encode abstract rules such as "choose the object that is the same" or "choose the object that is different" (Wallis et al., 2001a). More recently, prefrontal neurons have been shown to represent high-level behavioral strategies, such as a "repeat-stay" or "change-shift" strategy (Genovesio et al., 2005). Some of these neurons had strategy effects that were selective for a specific visual target, whereas others did not, suggesting that different levels of abstraction are coded in different sets of prefrontal neurons. In summary, these results implicate lateral prefrontal cortex in the maintenance of strategies, in addition to concrete behavioral rules.

\section{Conclusion}

Research on humans and nonhuman primates has led to the identification of a set of brain regions that mediate flexible ruleguided behavior. An important next step will be to characterize the temporal dynamics of interactions between these regions to gain additional insight into the neural mechanisms of rule use. Accordingly, studies involving simultaneous electrophysiological recordings at several sites would prove useful, as would a brain imaging technique with high spatial and temporal resolution, such as combined fMRI/electroencephalography. Additional research is also needed to explore the role of language in rule representation and to explore the neural basis of behavioral differences in rule use between species (Stoet and Snyder, 2003). An additional challenge will be to determine whether the distinctions drawn here between various types of rule representations are honored at the level of brain mechanisms. By studying how rules are retrieved from memory and used to guide action in specific situations, we will make progress in understanding the interface between perception, memory, and motor control.

\section{References}

Asaad WF, Rainer G, Miller EK (1998) Neural activity in the primate prefrontal cortex during associative learning. Neuron 21:1399-1407.

Bechara A, Damasio AR, Damasio H, Anderson SW (1994) Insensitivity to future consequences following damage to human prefrontal cortex. Cognition 50:7-15.

Bechara A, Damasio H, Tranel D, Anderson SW (1998) Dissociation of working memory from decision making within the human prefrontal cortex. J Neurosci 18:428-437.

Brass M, von Cramon DY (2002) The role of the frontal cortex in task preparation. Cereb Cortex 12:908-914.

Brass M, von Cramon DY (2004) Decomposing components of task preparation with functional magnetic resonance imaging. J Cogn Neurosci 16:609-620.

Brass M, Derrfuss J, Forstmann B, von Cramon DY (2005a) The role of the inferior frontal junction area in cognitive control. Trends Cogn Sci 9:314-316.

Brass M, Ullsperger M, Knoesche TR, von Cramon DY, Phillips NA (2005b) Who comes first? The role of the prefrontal and parietal cortex in cognitive control. J Cogn Neurosci, in press.

Bunge SA (2004) How we use rules to select actions: a review of evidence from cognitive neuroscience. Cogn Affect Behav Neurosci 4:564-579.

Bunge SA, Kahn I, Wallis JD, Miller EK, Wagner AD (2003) Neural circuits subserving the retrieval and maintenance of abstract rules. J Neurophysiol 90:3419-3428.

Crone EA, Wendelken C, Donohue S, Honomichl R, Bunge SA (2004) Contributions of prefrontal subregions to developmental changes in rule use. Soc Neurosci Abstr 30:253.10.

Crone EA, Wendelken C, Donohue SE, Bunge SA (2005) Evidence for sep- 
arable neural processes underlying flexible rule use. Cereb Cortex, in press.

Dias R, Robbins TW, Roberts AC (1996) Dissociation in prefrontal cortex of affective and attentional shifts. Nature 380:69-72.

Dreher JC, Berman KF (2002) Fractionating the neural substrate of cognitive control processes. Proc Natl Acad Sci USA 99:14595-14600.

Dum RP, Strick PL (2005) Frontal lobe inputs to the digit representations of the motor areas on the lateral surface of the hemisphere. J Neurosci 25:1375-1386.

Duvernoy HM (1991) The human brain: surface three-dimensional section anatomy and MRI. New York: Springer.

Eacott MJ, Gaffan D (1992) Inferotemporal-frontal disconnection: the uncinate fascicle and visual associative learning in monkeys. Eur J Neurosci 4:1320-1332.

Fellows LK, Farah MJ (2005) Different underlying impairments in decisionmaking following ventromedial and dorsolateral frontal lobe damage in humans. Cereb Cortex 15:58-63.

Fuster JM (2000) Executive frontal functions. Exp Brain Res 133:66-70.

Gaffan D, Easton A, Parker A (2002) Interaction of inferior temporal cortex with frontal cortex and basal forebrain: double dissociation in strategy implementation and associative learning. J Neurosci 22:7288-7296.

Genovesio A, Brasted PJ, Mitz AR, Wise SP (2005) Prefrontal cortex activity related to abstract response strategies. Neuron 47:307-320.

Hoshi E, Tanji J (2000) Integration of target and body-part information in the premotor cortex when planning action. Nature 408:466-470.

Hoshi E, Shima K, Tanji J (2000) Neuronal activity in the primate prefrontal cortex in the process of motor selection based on two behavioral rules. J Neurophysiol 83:2355-2373.

Izquierdo A, Murray EA (2004) Combined unilateral lesions of the amygdala and orbital prefrontal cortex impair affective processing in rhesus monkeys. J Neurophysiol 91:2023-2039.

Izquierdo A, Suda RK, Murray EA (2004) Bilateral orbital prefrontal cortex lesions in rhesus monkeys disrupt choices guided by both reward value and reward contingency. J Neurosci 24:7540-7548.

Kennerley SW, Lara AH, Wallis JD (2005) Prefrontal neurons encode an abstract representation of value. Soc Neurosci Abstr, in press.

Kim JN, Shadlen MN (1999) Neural correlates of a decision in the dorsolateral prefrontal cortex of the macaque. Nat Neurosci 2:176-185.

Kurata K, Wise SP (1988) Premotor cortex of rhesus monkeys: set-related activity during two conditional motor tasks. Exp Brain Res 69:327-343.

Leon MI, Shadlen MN (1999) Effect of expected reward magnitude on the response of neurons in the dorsolateral prefrontal cortex of the macaque. Neuron 24:415-425.

Lu MT, Preston JB, Strick PL (1994) Interconnections between the prefrontal cortex and the premotor areas in the frontal lobe. J Comp Neurol 341:375-392.

Matsumoto K, Suzuki W, Tanaka K (2003) Neuronal correlates of goalbased motor selection in the prefrontal cortex. Science 301:229-232.

Miller EK, Cohen JD (2001) An integrative theory of prefrontal cortex function. Annu Rev Neurosci 24:167-202.

Mitz AR, Godschalk M, Wise SP (1991) Learning-dependent neuronal activity in the premotor cortex: activity during the acquisition of conditional motor associations. J Neurosci 11:1855-1872.

Murray EA, Bussey TJ, Wise SP (2000) Role of prefrontal cortex in a network for arbitrary visuomotor mapping. Exp Brain Res 133:114-129.
Parker A, Gaffan D (1998a) Memory after frontal/temporal disconnection in monkeys: conditional and non-conditional tasks, unilateral and bilateral frontal lesions. Neuropsychologia 36:259-271.

Parker A, Gaffan D (1998b) Interaction of frontal and perirhinal cortices in visual object recognition memory in monkeys. Eur J Neurosci 10:3044-3057.

Passingham RE, Toni I, Rushworth MF (2000) Specialisation within the prefrontal cortex: the ventral prefrontal cortex and associative learning. Exp Brain Res 133:103-113.

Pasupathy A, Miller EK (2005) Different time courses of learning-related activity in the prefrontal cortex and striatum. Nature 433:873-876.

Roesch MR, Olson CR (2004) Neuronal activity related to reward value and motivation in primate frontal cortex. Science 304:307-310.

Rushworth MF, Ellison A, Walsh V (2001) Complementary localization and lateralization of orienting and motor attention. Nat Neurosci 4:656-661.

Rushworth MF, Hadland KA, Paus T, Sipila PK (2002) Role of the human medial frontal cortex in task switching: a combined fMRI and TMS study. J Neurophysiol 87:2577-2592.

Rushworth MF, Passingham RE, Nobre AC (2005) Components of attentional set-switching. Exp Psychol 52:83-98.

Sakai K, Passingham RE (2003) Prefrontal interactions reflect future task operations. Nat Neurosci 6:75-81.

Sohn MH, Goode A, Stenger VA, Carter CS, Anderson JR (2003) Competition and representation during memory retrieval: roles of the prefrontal cortex and the posterior parietal cortex. Proc Natl Acad Sci USA 100:7412-7417.

Stephens DW, Krebs JR (1986) Foraging theory. Princeton: Princeton UP.

Stoet G, Snyder LH (2003) Executive control and task-switching in monkeys. Neuropsychologia 41:1357-1364.

Stoet G, Snyder LH (2004) Single neurons in posterior parietal cortex of monkeys encode cognitive set. Neuron 42:1003-1012.

Tanji J, Hoshi E (2001) Behavioral planning in the prefrontal cortex. Curr Opin Neurobiol 11:164-170.

Tremblay L, Schultz W (2000) Modifications of reward expectation-related neuronal activity during learning in primate orbitofrontal cortex. J Neurophysiol 83:1877-1885.

Ungerleider LG, Gaffan D, Pelak VS (1989) Projections from inferior temporal cortex to prefrontal cortex via the uncinate fascicle in rhesus monkeys. Exp Brain Res 76:473-484.

Wallis JD, Miller EK (2003) Neuronal activity in primate dorsolateral and orbital prefrontal cortex during performance of a reward preference task. Eur J Neurosci 18:2069-2081.

Wallis JD, Anderson KC, Miller EK (2001a) Single neurons in prefrontal cortex encode abstract rules. Nature 411:953-956.

Wallis JD, Dias R, Robbins TW, Roberts AC (2001b) Dissociable contributions of the orbitofrontal and lateral prefrontal cortex of the marmoset to performance on a detour reaching task. Eur J Neurosci 13:1797-1808.

Watanabe M (1996) Reward expectancy in primate prefrontal neurons. Nature 382:629-632.

White IM, Wise SP (1999) Rule-dependent neuronal activity in the prefrontal cortex. Exp Brain Res 126:315-335.

Wise SP (1985) The primate premotor cortex: past, present, and preparatory. Annu Rev Neurosci 8:1-19. 